INPLASY

PROTOCOL

To cite: Chen et al. Qianzheng powder for the treatment of primary Hemifacial spasm A protocol for a systematic reviews and meta-analysis. Inplasy protocol 202130037. doi:

10.37766/inplasy2021.3.0037

Received: 13 March 2021

Published: 13 March 2021

Corresponding author:

Baoshan Chen

1164925816@qq.com

Author Affiliation: Jiangxi University of Traditional Chinese Medicine, Nanchang, China

Support: Jiangxi Province.

Review Stage at time of this submission: The review has not yet started.

Conflicts of interest: None declared.

\section{Qianzheng powder for the treatment of primary Hemifacial spasm A protocol for a systematic reviews and} meta-analysis

\author{
Chen, B1; A, R²; Yue, X33 Zhang, G4.
}

Review question / Objective: Can Qianzheng power improve quality of life in patients with Hemifacial spasm?

Condition being studied: Hemifacial spasm (HFS) is a clinical common neurological disease, its main performance for one side or two sides muscles (the orbicularis oculi muscle, expression, orbicularisoris muscles) recurrent paroxysmal, involuntary twitching, aggravating when excited or nervous, more severe cases of the disease may include difficulty in opening the eyes, crooked corners of the mouth, and twitching noises in the ears, etc. Early manifestations of the disease are intermittent mild convulsions of the orbicularis oculi muscle, and then gradually spread to one side of the facial muscles, such as frowning muscles, nasal muscles, buccinalis muscles, etc, especially the most obvious spasms of the oral muscles, which can involve the ipsilateral platysma muscle in severe cases, with each twitch for a few seconds to a few minutes. The disease will affect the quality of life such as speaking, eating, seeing and so on, and even cause psychological effects such as inferiority, anxiety and depression. At present, the incidence of the disease in China is $\mathbf{1 1}$ per 1.1 million, females are more common than males.There are many ways to treat HFS, but the Qianzheng powder has a unique advantage in treating this disease. Therefore, our systematic review aims to evaluate the efficacy and safety.

INPLASY registration number: This protocol was registered with the International Platform of Registered Systematic Review and Meta-Analysis Protocols (INPLASY) on 13 March 2021 and was last updated on 13 March 2021 (registration number INPLASY202130037).

\section{INTRODUCTION}

Review question / Objective: Can Qianzheng power improve quality of life in patients with Hemifacial spasm?
Condition being studied: Hemifacial spasm (HFS) is a clinical common neurological disease, its main performance for one side or two sides muscles (the orbicularis oculi 
muscle, expression, orbicularisoris muscles) recurrent paroxysmal, involuntary twitching, aggravating when excited or nervous, more severe cases of the disease may include difficulty in opening the eyes, crooked corners of the mouth, and twitching noises in the ears, etc. Early manifestations of the disease are intermittent mild convulsions of the orbicularis oculi muscle, and then gradually spread to one side of the facial muscles, such as frowning muscles, nasal muscles, buccinalis muscles, etc, especially the most obvious spasms of the oral muscles, which can involve the ipsilateral platysma muscle in severe cases, with each twitch for a few seconds to a few minutes. The disease will affect the quality of life such as speaking, eating, seeing and so on, and even cause psychological effects such as inferiority, anxiety and depression. At present, the incidence of the disease in China is 11 per 1.1 million, females are more common than males. There are many ways to treat HFS, but the Qianzheng powder has a unique advantage in treating this disease. Therefore, our systematic review aims to evaluate the efficacy and safety.

\section{METHODS}

Participant or population: Participants are patients with Hemifacial spasm.

Intervention: Qianzheng powder as a single intervention means, there is no limit to the dosage, addition or subtraction of the medication, or in combination with other intervention measures (such as conventional drugs, acupuncture, moxibustion, etc.).

Comparator: The control group had no limits on treatment, including conventional drugs, no treatment, or placebo.

Study designs to be included: Randomized controlled trials.

Eligibility criteria: Randomised controlled trials evaluating Qianzheng powder in the treatment of primary HFS will be eligible for inclusion and will be published in English or
Chinese, with the full-text available. Patients with primary HFS with a clear diagnostic criteria will be included.

Information sources: We will search electronic databases, including PubMed, Embase, Cochrane Library, China Biomedical Literature Database (CBM), China National Knowledge Infrastructure (CNKI), Wanfang Database (WF), and China Science Journals Database (VIP), to collect potential RCTs from the start through April 2021.If there are any problems with the study, we will try to contact the author.

Main outcome(s): Total effective rate (assessed based on Cohen criteria for efficacy evaluation of Hemifacial spasm, total effective rate $=($ cure + significant effect + effective)/total).

Quality assessment / Risk of bias analysis: The bias risk assessment tool recommended by the Cochrane Collaboration Network was used to assess the quality of the included studies. The following seven evaluation items are included: (1)random sequence generation; (2)allocation concealment; (3)blinding of participants and personnel; (4)blinding of outcome assessment; (5)incomplete outcome data; (6)selective reporting; (7)other sources of bias For each study, the results of the seven items were rated as "Yes" (low risk), "No" (high risk) and "unclear" (lack of information or uncertainty about bias). Two reviewers independently conduct quality assessments and any differences will be resolved through discussion.

Strategy of data synthesis: We will use RevMan V. 5.3 software for statistical analysis. For dichotomous variables, relative risk (RR) and $95 \%$ confidence intervals $(\mathrm{Cl})$ were used.

Subgroup analysis: If significant heterogeneity is detected in our metaanalysis, we will perform subgroup analysis according to different control groups.

Sensitivity analysis: When sufficient RCTs are available, we will conduct sensitivity 
analysis based on method quality, sample size, and missing data to assess the robustness of the study results.

Language: Chinese and English.

Country(ies) involved: China.

Keywords: Primary Hemifacial spasm, Qianzheng powder, protocol, systematic review.

Contributions of each author:

Author 1 - Baoshan Chen.

Email: 1164925816@qq.com

Author 2 - Rigun A.

Email: 389014066@qq.com

Author 3 - Ruizhen Yue.

Email: 958499575@qq.com

Author 4 - Guangrong Zhang.

Email: zhgr321@qq.com 